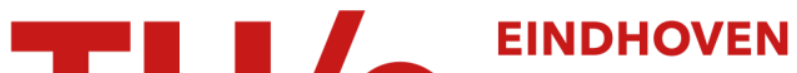

\section{Allocation of excitation signals for generic identifiability of dynamic networks}

\section{Citation for published version (APA):}

Cheng, X., Shi, S., \& Van Den Hof, P. M. J. (2019). Allocation of excitation signals for generic identifiability of dynamic networks. In 2019 IEEE 58th Conference on Decision and Control, CDC 2019 (pp. 5507-5512).

[9029218] (Proceedings of the IEEE Conference on Decision and Control; Vol. 2019-December). Institute of Electrical and Electronics Engineers. https://doi.org/10.1109/CDC40024.2019.9029218

DOI:

10.1109/CDC40024.2019.9029218

Document status and date:

Published: 01/12/2019

\section{Document Version:}

Accepted manuscript including changes made at the peer-review stage

\section{Please check the document version of this publication:}

- A submitted manuscript is the version of the article upon submission and before peer-review. There can be important differences between the submitted version and the official published version of record. People interested in the research are advised to contact the author for the final version of the publication, or visit the $\mathrm{DOI}$ to the publisher's website.

- The final author version and the galley proof are versions of the publication after peer review.

- The final published version features the final layout of the paper including the volume, issue and page numbers.

Link to publication

\section{General rights}

Copyright and moral rights for the publications made accessible in the public portal are retained by the authors and/or other copyright owners and it is a condition of accessing publications that users recognise and abide by the legal requirements associated with these rights.

- Users may download and print one copy of any publication from the public portal for the purpose of private study or research.

- You may not further distribute the material or use it for any profit-making activity or commercial gain

- You may freely distribute the URL identifying the publication in the public portal.

If the publication is distributed under the terms of Article 25fa of the Dutch Copyright Act, indicated by the "Taverne" license above, please follow below link for the End User Agreement:

www.tue.nl/taverne

Take down policy

If you believe that this document breaches copyright please contact us at:

openaccess@tue.nl

providing details and we will investigate your claim. 


\title{
Allocation of Excitation Signals for Generic Identifiability of Dynamic Networks
}

\author{
Xiaodong Cheng, Shengling Shi, and Paul M.J. Van den Hof
}

\begin{abstract}
This paper studies generic identifiability of dynamic networks, in which the edges connecting the vertex signals are described by proper transfer functions, and partial vertices are excited by designed external signals. We assume that the topology of the underlying graph is known, and all the vertex signals are measured. We show that generic identifiability of a directed network is related to the existence of a set of disjoint directed pseudo-trees that cover all the edges of the underlying graph, based on which, an excitation allocation problem is studied, aiming to select the minimal number excitation signals to achieve the generic identifiability of the whole network. An algorithmic procedure thereby is devised for selecting locations of the external signals such that all the edges can be consistently estimated.
\end{abstract}

\section{INTRODUCTION}

Dynamic networks can adequately describe a wide class of complex engineering systems, which appear in various applications, including multi-robot coordination and distributed control of power grids [1], [2]. The conventional system identification mainly focuses on the systems with relatively simple dynamical structures, e.g., single-input-single-output (SISO), multiple-input-multiple-output (MIMO), open-loop or closed-loop systems [3], [4]. However, these classical data-driven tools seem to be limited when encountering dynamic networks with complex interconnection structures. The bridge connecting the classic identification framework and dynamic networks is initially built in [5], where the vertices in a network are interpreted as measured internal signals, and the directed edges represent transfer operators, referred as modules. Considering external noises and excitation signals, the identification of the modules in a network can be recast as a closed-loop system identification problem.

Based on this setup, three problems have been addressed. The first is to detect the topology of a network, see e.g., [6], [7], where techniques, such as Wiener filters or Bayesian approaches are taken to obtain sparse estimates. The second problem is to estimate a desired local module within a network, see e.g., [8]-[11], which focus on the question: under what conditions we are able to consistently identify the dynamics of a selected module in the network? In contrast, the problem in this paper concerns the structural identifiability of a full dynamic network, see e.g., [12]-[15] and the references therein. Assuming that the topology of a

This work is supported by the European Research Council (ERC), Advanced Research Grant SYSDYNET, under the European Unions Horizon 2020 research and innovation programme (Grant Agreement No. 694504).

The authors are with Control Systems Group, Department of Electrical Engineering, Eindhoven University of Technology, 5600 MB Eindhoven, The Netherlands. \{x.cheng, s.shi, P.M.J.vandenhof \} @tue.nI network is known a priori, a model set is considered in which all the models are associated with the given topology. Then identifiability essentially reflects the ability to distinguish between these models on the basis of measurement data.

In the literature, there are two classes of network identifiability, namely, global identifiability [13], [15] that requires all the models in the set to be distinguishable, and generic identifiability [14], [16], which means that almost all models in the model set can be distinguished. In the study of identifiability, two problem settings of dynamic networks are considered. In e.g., [14], [15], all vertices are excited by external signals, while only a subset of vertices is measured, and in e.g., [13], [16], all internal variables are supposed to be measured, while only partial vertices are excited or influenced by noises. In this paper, the generic identifiability is of particular interest. Given the topology of a network consisting of internal signals, we aim for a systematic scheme that finds the minimum number of excitation signals for the generic identifiability of a dynamic network. To the best of our knowledge, such a design problem has not been addressed in the context of generic identifiability for dynamic networks.

Inspired by [13], [14], we analyze the generic identifiability from a graph-theoretic point of view and provide a new characterization using the concept of disjoint pseudotree covering. It is shown that a directed dynamic network is generically identifiable if there exist a set of disjoint pseudo-trees covering all the edges of the underlying graph, and the external signals can be allocated at the roots of these pseudo-trees. Thereby, we propose a graph merging approach, which first partitions the network into several minimal disjoint pseudo-trees and then iteratively aggregates pairs that are mergeable. The merging process is consistent with the operation on the characterization matrix of the disjoint pseudo-tree covering.

The rest of this paper is organized as follows: In Section II, we recap some basic notations in graph theory and introduce the network model. Section III then presents a graphtheoretic approach to the allocation of excitation signals, and finally, concluding remarks are made in Section IV.

Notation: Denote $\mathbb{R}$ as the set of real numbers, and $\mathbb{R}(q)$ is the rational function field over $\mathbb{R}$ with variable $q . A_{i j}$ denotes the $(i, j)$-th entry of a matrix $A$. The cardinality of a set $\mathcal{V}$ is given by $|\mathcal{V}|$. Let $\mathcal{G}$ be a directed graph, and we denote $V(\mathcal{G})$ and $E(\mathcal{G})$ as the vertex set and edge set of $\mathcal{G}$, respectively. The union of two graphs $\mathcal{G}_{1}$ and $\mathcal{G}_{2}$ is denoted by $\mathcal{G}:=\mathcal{G}_{1} \cup \mathcal{G}_{2}$, where $V(\mathcal{G})=V\left(\mathcal{G}_{1}\right) \cup V\left(\mathcal{G}_{2}\right)$ and $E(\mathcal{G})=E\left(\mathcal{G}_{1}\right) \cup E\left(\mathcal{G}_{2}\right)$. 


\section{PRELIMINARIES}

\section{A. Graph theory}

We provide necessary terminologies and concepts from graph theory and refer to [1], [17] for more details. The topology of a network is characterized by a graph $\mathcal{G}$ that consists of a finite and nonempty vertex set $\mathcal{V}:=\{1,2, \cdots, L\}$ and an edge set $\mathcal{E} \subseteq \mathcal{V} \times \mathcal{V}$. For a directed graph, each element in $\mathcal{E}$ is an ordered pair of elements of $\mathcal{V}$, and if $(i, j) \in \mathcal{E}$, we say that the edge is incident from vertex $i$ to vertex $j$. The vertex $i$ is the in-neighbor of $j$, and $j$ is the out-neighbor of $i$. Let $\mathcal{N}_{i}^{-}$and $\mathcal{N}_{i}^{+}$be the sets that collect all the in-neighbors and out-neighbors of vertex $i$, respectively. Let $S$ be the set that collects all sinks of $\mathcal{G}$, namely,

$$
S(\mathcal{G}):=\left\{i \in V(\mathcal{G})|| \mathcal{N}_{i}^{+} \mid=0\right\} .
$$

A graph $\mathcal{G}$ is called simple, if $\mathcal{G}$ does not contain selfloops (i.e., $\mathcal{E}$ does not contain edges of the form $(i, i), \forall i$ ), and there exists only one directed edge from one vertex to its each out-neighbor. A directed path in a simple graph connecting vertices $i_{0}$ and $i_{n}$ is a sequence of edges of the form $\left(i_{k-1}, i_{k}\right), k=1, \cdots, n$, and every vertex appears at most once on the path. Two directed paths are vertex disjoint if they do not share any common vertex, including the start and the end vertices. A connected directed simple graph $T$ is a directed tree (or an arborescence), if there exists a vertex $r$, known as the root vertex, from which there is exactly one directed path from $r$ to every other vertices in $T$. A vertex $i$ in a tree $T$ is called a leaf if $\left|\mathcal{N}_{i}^{+}\right|=0$, and the vertices that are neither the root nor leaves of $T$ are called the internal vertices of $T$. In a rooted tree, a vertex $i$ is a child of vertex $j$ if there is a directed edge incident from $j$ to $i$, in which case, vertex $j$ is the (only) parent of $i$.

\section{B. Dynamic network model}

Consider a simple directed graph $\mathcal{G}=(\mathcal{V}, \mathcal{E})$ with vertex set $\mathcal{V}=\{1,2, \cdots, L\}$ and edge set $\mathcal{E} \subseteq \mathcal{V} \times \mathcal{V}$. Then, following the basic setup of [5], [13], a dynamic network associated with $\mathcal{G}$ is defined as follows.

$$
w(t)=G(q) w(t)+R(q) r(t),
$$

where $q^{-1}$ is the delay operator, i.e. $q^{-1} w_{j}(t)=w_{j}(t-1)$. $w(t) \in \mathbb{R}^{L}$ and $r(t) \in \mathbb{R}^{K}$ are vectors of measured internal signals and external excitation signals, respectively. Let $\mathcal{R} \subseteq$ $\mathcal{V}$ be the set of vertices that are excited by the external signals $r(t)$, and $K:=|\mathcal{R}|$. Each vertex in $\mathcal{R}$ is called a excited vertex. Throughout the paper, the transfer matrices $G(q), R(q)$ satisfy the following properties [5], [13].

- All the entries of $G(q)$ are strictly proper transfer functions to be identified (i.e., parameterized), and the entries of $R(q)$ are known proper transfer functions.

- Each row and each column of $R(q)$ contain only one nonzero entry, i.e., each vertex in $\mathcal{R}$ is influenced by a unique external excitation signal.

- The network $\mathcal{G}$ is well-posed, i.e., $(I-G(q))^{-1}$ is proper and stable. Moreover, $R_{i k}(q)$ is stable.
In this paper, we are interested in the condition under which all the transfer functions in $G(q)$ can be consistently identified from the external excitation signals $r(t)$ and the measurement data $w(t)$. Thereby, we assume that the topology is known a priori, and all the vertex signals $w(t)$ are measured. Then, we aim to allocate a minimal number of external excitation signals such that the full network is generically identifiable.

\section{MAIN RESULTS}

\section{A. Generic identifiability: a graph-theoretical condition}

Identifiability is basically the ability to uniquely identify transfer functions in $G(q)$ from excitation signals $r(t)$ and the measurement date $w(t)$. Following [13], we denote a set of parameterized matrix-valued functions

$$
\Sigma:=\{\sigma(q, \theta)=(G(q, \theta), R(q)), \theta \in \Theta\}
$$

as the network model set for a network described in (2), where $G(q, \theta)$ is a strictly proper transfer function for all $\theta \in \Theta$. Let

$$
T(q, \theta)=(I-G(q, \theta))^{-1} R(q)
$$

Then, the generic identifiability of $\Sigma$ is defined as follows.

Definition 1 (Generic identifiability): The network model set $\Sigma$ is generically identifiable if the implication

$$
T\left(q, \theta_{1}\right)=T\left(q, \theta_{0}\right) \Rightarrow \sigma\left(q, \theta_{1}\right)=\sigma\left(q, \theta_{0}\right),
$$

holds for all parameters $\theta_{1}, \theta_{0} \in \Theta$ except possibly those lying on a zero measure set in $\Theta$.

We refer to e.g., [14], [16] for more details on the definition of generic identifiability. The generic identifiability of every module $G_{j i}$ is implied by the model set $\Sigma$ being generically identifiable. If all the modules are generically identifiable, we then say the dynamic network $\mathcal{G}$ is generically identifiable. In the following lemma, a graph-theoretic condition has been studied for checking generic identifiability.

Lemma 1: [14], [16] A dynamic network is generically identifiable if and only if the maximum number of mutually vertex disjoint paths from $\mathcal{R}$ to $\mathcal{N}_{i}^{-}$is greater than or equal to $\left|\mathcal{N}_{i}^{-}\right|$for all $i \in \mathcal{V}$.

Note that the characterization of the generic identifiability in Lemma 1 relates to all the vertex disjoint paths from the excited vertices (i.e., the vertices in $\mathcal{R}$ ) to the in-neighbors of each vertex in a network. For the allocation problem studied in the current paper, the use of the condition in Lemma 1 may be restricted, as it requires a path check of each vertex in the network. In contrast, this paper proposes a more integrated graph-theoretic condition for characterizing the generic identifiability.

Before proceeding, the concept of directed pseudo-trees is introduced.

Definition 2 (Directed pseudo-trees): A connected directed graph $\mathcal{T}$ is called a (directed) pseudo-tree if $\left|\mathcal{N}_{i}^{-}\right| \leq 1$, for all $i \in V(\mathcal{T})$.

The above concept of pseudo-trees is an extension of its definition in the undirected case, in which they are 


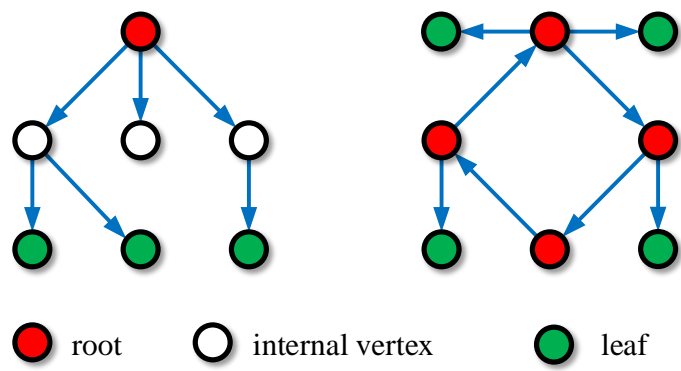

Fig. 1. Typical examples of pseudotrees, in which roots, internal vertices and leaves are labeled with different colors. Note that a pseudotree may have multiple roots.

also referred to as unicyclic graphs, see e.g., [18], [19]. Analogous to directed tree graphs, we have the following definitions:

- A vertex in the directed pseudo-tree $\mathcal{T}$ a root of $\mathcal{T}$, if there is exactly one directed path from this vertex to every other vertices in $\mathcal{T}$;

- A vertex in $\mathcal{T}$ is a leaf of $\mathcal{T}$, if it has no out-neighbors in $\mathcal{T}$;

- A vertex in $\mathcal{T}$ that is neither a root nor a leaf is an internal vertex of $\mathcal{T}$.

The above definitions are illustrated in Fig. fig:pseudotrees, where two typical directed pseudo-trees are shown. Furthermore, we call a directed pseudo-tree minimal if it only contains one root and all the out-neighbors of the root as leaves. It is remarked that directed pseudo-trees allow for multiple roots, and any directed pseudo-tree can be expressed as a union of a directed tree with a possible directed edge incident from a leaf or an internal vertex of the tree to its root. Thus, directed pseudo-trees are more general definition than directed trees. Hereafter, we will drop the word 'directed' when we refer to a directed pseudo-tree, and denote $\Upsilon(\mathcal{T})$ as a set that collects all the roots of the pseudo-tree $\mathcal{T}$. Related to the concept of vertex disjoint paths, disjoint directed pseudo-trees are defined.

Definition 3 (Disjoint pseudo-trees): Consider two pseudo-trees $\mathcal{T}_{1}$ and $\mathcal{T}_{2}$ as subgraphs of a directed graph $\mathcal{G} . \mathcal{T}_{1}$ and $\mathcal{T}_{2}$ are called disjoint in $\mathcal{G}$ if the following two conditions hold.

1) $E\left(\mathcal{T}_{1}\right) \cap E\left(\mathcal{T}_{2}\right)=\emptyset$;

2) For each $j \in V\left(\mathcal{T}_{1}\right) \cup V\left(\mathcal{T}_{2}\right)$, all the edges in $V E\left(\mathcal{T}_{1}\right) \cup$ $E\left(\mathcal{T}_{2}\right)$ that are incident from $j$ are included in $E\left(\mathcal{T}_{1}\right)$ or $E\left(\mathcal{T}_{2}\right)$.

The first condition means that disjoint pseudo-trees do not share common edges, and the second condition requires that all the edges incident from each vertex should be included in one pseudo-tree. As a special case, if both $\mathcal{T}_{1}$ and $\mathcal{T}_{2}$ are directed rooted trees, then $\mathcal{T}_{1}$ and $\mathcal{T}_{2}$ do not share the same root or any common internal vertex. We illustrate the concept of disjoint pseudo-trees with the following example.

Example 1: In Fig. 1, to illustrate disjoint pseudo-trees in a directed graph, we decompose two graphs in (a) and (b) into two pseudo-trees. By Definition 3, the two pseudo-trees

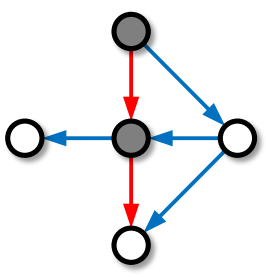

(a)

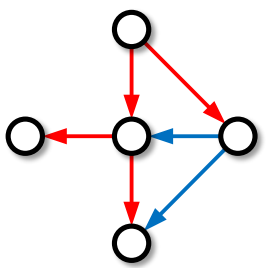

(c)

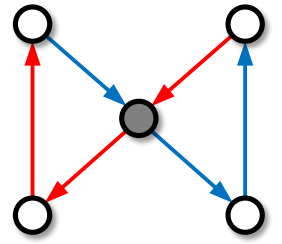

(b)

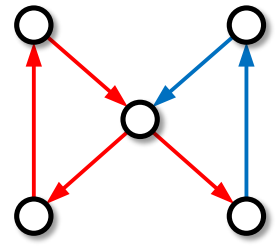

(d)
Fig. 2. Illustration of disjoint pseudo-trees, in which different pseudo-trees are induced by the edges with distinct colors. In (a) and (b), the pseudotrees are not disjoint, since the outgoing edges from the gray vertices are assigned to different pseudo-trees. In contrast, the pseudo-trees in (c) and (d) are considered to be disjoint pairs.

in both (a) and (b) are not disjoint. In (a), the two trees share the same root and an internal vertex in the center. In (b), the gray vertex in the center violate the second condition in Definition 3, as its two outgoing edges are included in different pseudotrees. If we take different decompositions of the networks, the two pseudotrees obtained in (c) and (d) are shown to be disjoint.

Next, the concept of disjoint-edge covering for a directed graph is introduced.

Definition 4 (Disjoint-edge covering): Consider a directed graph $\mathcal{G}$, and let $\mathcal{T}_{1}, \mathcal{T}_{2}, \cdots, \mathcal{T}_{K}$ be a collection of connected subgraphs of $\mathcal{G}$. The edges of $\mathcal{G}$ are covered by $\mathcal{T}_{1}, \mathcal{T}_{2}, \cdots, \mathcal{T}_{K}$ if $E\left(\mathcal{T}_{1}\right) \cup E\left(\mathcal{T}_{2}\right) \cup \cdots \cup E\left(\mathcal{T}_{K}\right)=E(\mathcal{G})$ The set $\Pi:=\left\{\mathcal{T}_{1}, \mathcal{T}_{2}, \cdots, \mathcal{T}_{K}\right\}$ is called a covering of $\mathcal{G}$. Moreover. $\Pi$ is disjoint pseudo-tree covering, if all the elements in $\Pi$ are pseudo-trees, which are disjoint to each other.

With the definition of the disjoint pseudo-tree covering, the following lemma is given.

Lemma 2: For any directed network $\mathcal{G}$, there always exists a set of disjoint pseudo-trees that cover all the edges of $\mathcal{G}$.

Proof: For any directed graph $\mathcal{G}$, a set of disjoint pseudo-trees can be constructed as follows. For each vertex $k \in V(\mathcal{G})-S(\mathcal{G})$, with $S(\mathcal{G})$ the set of sinks, we construct a directed star tree (i.e., a minimal pseudo-tree) with $k$ as the root and the vertices in $\mathcal{N}_{k}^{+}$as leaves. Then, $|V(\mathcal{G})-S(\mathcal{G})|$ minimal pseudo-trees are formed, which are disjoint, since any two trees do not share a common root or any common internal vertex.

Now, we are ready present the main conclusion.

Theorem 1: Consider a directed network $\mathcal{G}$, and let $\mathcal{R}=$ $\left\{\tau_{1}, \tau_{2}, \cdots, \tau_{K}\right\} \subseteq V(\mathcal{G})$ be the set of excited vertices. Then, the network $\mathcal{G}$ is generically identifiable if there exists a disjoint pseudo-tree covering of $\mathcal{G}$, denoted by 
$\Pi=\left\{\mathcal{T}_{1}, \mathcal{T}_{2}, \cdots, \mathcal{T}_{K}\right\}$ such that $\tau_{i} \in \Upsilon\left(\mathcal{T}_{i}\right)$, for all $i \in$ $\{1,2, \cdots, K\}$.

Proof: Assume that there exist a disjoint pseudo-tree covering, $\Pi=\left\{\mathcal{T}_{1}, \mathcal{T}_{2}, \cdots, \mathcal{T}_{K}\right\}$, and in each pseudo-tree $\mathcal{T}_{k}$, one of its root vertices is excited by an external signal $r_{k}(t)$, i.e., $\tau_{i} \in \Upsilon\left(\mathcal{T}_{k}\right)$. Since there does not exist any two vertices in a same pseudo-tree sharing a common child, for any vertex $i \in V(\mathcal{G})$, all the edges incident from vertices in $\mathcal{N}_{i}^{-}$to $i$ should belong to different pseudo-trees, and $\left|\mathcal{N}_{i}^{-}\right| \leq K$ holds. Thus, there exist at least $\left|\mathcal{N}_{i}^{-}\right|$vertex disjoint paths from $\left\{\tau_{1}, \tau_{2}, \cdots, \tau_{K}\right\}$ to $\mathcal{N}_{i}^{-}$. As each $\tau_{i}$, a root of a pseudo-tree $\mathcal{T}_{i}$, is excited, the generic identifiability of $\mathcal{G}$ then immediately follows from Lemma 1.

The following result is obtained by directly applying Theorem 1.

Corollary 1: The minimal number of external signals that are needed for the generic identifiability of a directed graph $\mathcal{G}$ is upper-bounded by the minimal number of disjoint pseudotrees that cover all the edges of $\mathcal{G}$.

\section{B. Excitation allocation: a graph merging approach}

In this section, we aim to solve an excitation allocation problem, which aims for a minimal number of external excitation signals which are used to consistently estimate the strictly proper transfer functions associated with all the edges of a network.

Problem 1: Given a directed network $\mathcal{G}$, find $\mathcal{R} \subseteq V(\mathcal{G})$ as the set of excited vertices such that $|\mathcal{R}|$ is minimized, and $\mathcal{G}$ is generic identifiable.

Note that finding the exact minimal number of the excitation signals and their locations is difficult in general. The previous subsection shows that the upper bound of this number actually relates the disjoint pseudo-tree covering. Then, to solve the above problem, we find the minimal number of disjoint pseudo-trees, which are not rooted at $\mathcal{R}$ in the network, such that all the edges are covered. At this point, Problem 1 is converted to a combinatorial optimization problem. In this section, we devise an algorithmic procedure to partition a graph into disjoint pseudo-trees with as less number as possible.

Lemma 2 indicates that for any directed graph $\mathcal{G}$, we can always find a disjoint pseudo-tree covering,

$$
\Pi_{0}=\left\{\mathcal{T}_{1}^{0}, \mathcal{T}_{2}^{0}, \cdots, \mathcal{T}_{\left|\Pi_{0}\right|}^{0}\right\},
$$

in which each element is a minimal pseudo-tree rooted at a vertex that is not a sink. We start with $\Pi_{0}$ as our initial disjoint pseudo-tree covering, and the strategy is to recursively merge the pseudo-trees until there are no mergeable pseudo-trees in a covering. Specifically, the mergeability of pseudo-trees are defined as follows.

Definition 5 (Mergeability): Consider two disjoint pseudo-trees $\mathcal{T}_{1}$ and $\mathcal{T}_{2}$. We say $\mathcal{T}_{1}$ is mergeable to $\mathcal{T}_{2}$, if

1) the union of $\mathcal{T}_{1}$ and $\mathcal{T}_{2}$, i.e., $\left(V\left(\mathcal{T}_{1}\right) \cup V\left(\mathcal{T}_{2}\right), E\left(\mathcal{T}_{1}\right) \cup\right.$ $\left.E\left(\mathcal{T}_{2}\right)\right)$ is also a pseudo-tree;

2) there is a directed path from vertex $i$ to vertex $j$, for all $i \in \Upsilon\left(\mathcal{T}_{2}\right)$ and $j \in V\left(\mathcal{T}_{1}\right)$.
The mergeability from a pseudo-tree $\mathcal{T}_{1}$ to $\mathcal{T}_{2}$ requires that $\mathcal{T}_{1}$ and $\mathcal{T}_{2}$ does not share any common leaf and internal vertex. As a result, merging $\mathcal{T}_{1}$ and $\mathcal{T}_{2}$ yields a new pseudo-tree $\mathcal{T}_{3}$, where $\Upsilon\left(\mathcal{T}_{2}\right) \subseteq \Upsilon\left(\mathcal{T}_{3}\right)$. Note that $\mathcal{T}_{1}$ being mergeable to $\mathcal{T}_{2}$ does not necessarily mean that $\mathcal{T}_{2}$ is also mergeable to $\mathcal{T}_{1}$.

Given a disjoint pseudo-tree covering of $\mathcal{G}$. We introduce a characteristic matrix $\mathcal{M}$, whose $(i, j)$-th entry is defined as follows.

$$
\mathcal{M}(i, j)= \begin{cases}1 & \text { if } \mathcal{T}_{i} \text { is mergeable to } \mathcal{T}_{j} \\ \varnothing & \text { if } V\left(\mathcal{T}_{i}\right) \cap V\left(\mathcal{T}_{j}\right)=\emptyset \\ 0 & \text { otherwise }\end{cases}
$$

Related to the characteristic matrix, the following notations and operations are defined. Let $\mathcal{M}(i,:)$ and $\mathcal{M}(:, j)$ be the $i$-th row and $j$-th column of a matrix $\mathcal{M} \in \mathbb{M}$, and denote

$$
\mathbb{M}:=\{1, \varnothing, 0\}
$$

Then we have $\mathcal{M} \in \mathbb{M}^{|\Pi| \times|\Pi|}$, where $\Pi$ is a disjoint pseudotree covering. We define $c=a \odot b=b \odot a$, with $a, b, c \in \mathbb{M}$, as a commutative operation, which follows the rules:

$$
\begin{aligned}
& 1 \odot 1=1,1 \odot 0=0,1 \odot \varnothing=1, \\
& 0 \odot 0=0, \varnothing \odot 0=0, \varnothing \odot \varnothing=\varnothing .
\end{aligned}
$$

Furthermore, let $\rho, \mu$ be two column (or row) vectors of the same dimensions in $\mathbb{M}$. Then, $\rho \odot \mu=\mu \odot \rho$ stands for an entrywise operation that returns a new column (or row) vector, whose $i$-th element is given by $\rho_{i} \odot \mu_{i}$.

For a given disjoint pseudo-tree covering $\Pi$ with $|\Pi|=$ $n$ and a set $\mathbb{N}:=\{1,2, \cdots, n\}$, we define the following function

$$
\mathscr{F}: \mathbb{M}^{n \times n} \times \mathbb{N} \times \mathbb{N} \rightarrow \mathbb{M}^{(n-1) \times(n-1)},
$$

and $\hat{\mathcal{M}}=\mathscr{F}(\mathcal{M}, i, j)$ is a reduction of $\mathcal{M}$ obtained by the following algebraic operations:

1) $\hat{\mathcal{M}}=\mathcal{M}$

2) Row merging: $\hat{\mathcal{M}}(i,:)=\mathcal{M}(i,:) \odot \mathcal{M}(j,:)$;

3) Column merging: $\hat{\mathcal{M}}(:, i)=\mathcal{M}(:, i) \odot \mathcal{M}(:, j)$;

4) Remove $j$-th row and column of $\hat{\mathcal{M}}$.

The operation $\mathscr{F}(\mathcal{M}, i, j)$ means that $\mathcal{T}_{i}$ is merged to $\mathcal{T}_{j}$. It is also worth emphasizing that the order of the row and column operations can be switched, which will not affect the outcome $\hat{\mathcal{M}}$. Furthermore, reducing the characteristic matrix is consistent with the change in the disjoint pseudotree covering of the network. Specifically, if in $\Pi$, a pseudotree $\mathcal{T}_{i}$ is mergeable to $\mathcal{T}_{j}$, then $\mathscr{F}(\mathcal{M}, i, j)$ produces a $(|\Pi|-1) \times(|\Pi|-1)$ characteristic matrix $\hat{\mathcal{M}}$ representing a new disjoint pseudo-tree covering $\hat{\Pi}$, which contains one less element compared to $\Pi$.

The foundation of the merging algorithm has been laid above. Now we present a greedy scheme, aiming to find a disjoint pseudo-tree covering with the minimal number of elements.

Step 1: For the initial disjoint pseudo-tree covering $\Pi=$ $\Pi_{0}$ in (5), we obtain its characteristic matrix $\mathcal{M}$. 
Step 2: Find an entry $\mathcal{M}(i, j)=1$ such that the $i$-th row contains only one single "1" entry, while the others are either " 0 " or " $\varnothing$ ". If there exist multiple rows containing a single " 1 " entry, then we choose the one with more " $\varnothing$ " entries. Then, we merge $\mathcal{T}_{i}$ to $\mathcal{T}_{j}$, and update $\mathcal{M}$ as $\mathcal{M} \leftarrow \mathscr{F}(\mathcal{M}, i, j)$. We repeat this step until there is no row containing only one single " 1 " entry.

Step 3: We find an entry $\mathcal{M}(i, j)=1$ such that the $i$-th row with the most " $\varnothing$ " entries. Then, we merge $\mathcal{T}_{i}$ to $\mathcal{T}_{j}$, and update $\mathcal{M}$ as $\mathcal{M} \leftarrow \mathscr{F}(\mathcal{M}, i, j)$. We repeat this step until there is no row containing " 1 " entries.

We further explain the above steps. In the first step, we merge $\mathcal{T}_{i}$ to $\mathcal{T}_{j}$, if $\mathcal{T}_{j}$ is the only pseudo-tree that $\mathcal{T}_{i}$ is mergeable to. If there are multiple pairs satisfying this condition, we then select the one that has more nonoverlapping pseudo-trees. Two disjoint pseudo-trees are nonoverlapping if they do not share any vertex in common. By doing so, the current merging will cause less effect on the follow-up merging. A subsequent operation in Step 2 follows a similar mechanism and merge the left mergeable pseudotrees together.

The number of the pseudo-trees in the final covering is the number of excitation signals that are designed to stimulate the network for generic identifiability. The locations of these excitation signals can be assigned to distinct vertices that are the roots of the generated disjoint pseudo-trees. We have to emphasize that the presented algorithm is a greedy approach and does not always provide a minimal set of pseudo-trees. However, the presented algorithm gives an effective solution to allocate a smaller number of excitation signals for the generic identifiability of a given dynamic network. In the following subsection, we illustrate the steps of the algorithm using an simple network example.

\section{Case Study}

Consider a directed simple graph with 10 vertices, as shown in Fig. 3. An initial disjoint pseudo-tree covering is given as $\Pi_{0}=\left\{\mathcal{T}_{1}^{0}, \mathcal{T}_{2}^{0}, \cdots, \mathcal{T}_{9}^{0}\right\}$, in which each pseudotree has a single root vertex, which is not a sink of $\mathcal{G}$. By the definition in (6), we construct the following matrix for characterizing the mergeability.

$$
\mathcal{M}_{0}=\left[\begin{array}{lllllllll}
0 & 1 & \varnothing & \varnothing & 0 & \varnothing & 0 & \varnothing & \varnothing \\
0 & 0 & 1 & \varnothing & 0 & 0 & 0 & \varnothing & \varnothing \\
\varnothing & 1 & 0 & 0 & \varnothing & 0 & \varnothing & 0 & 0 \\
\varnothing & \varnothing & 0 & 0 & \varnothing & 0 & \varnothing & 0 & 0 \\
0 & 1 & \varnothing & \varnothing & 0 & 1 & 0 & 0 & \varnothing \\
\varnothing & 0 & 1 & 1 & 0 & 0 & 0 & 0 & 1 \\
0 & 0 & \varnothing & \varnothing & 0 & 0 & 0 & 1 & \varnothing \\
\varnothing & \varnothing & 0 & 0 & 1 & 0 & 0 & 0 & 0 \\
\varnothing & \varnothing & 0 & 0 & \varnothing & 0 & \varnothing & 0 & 0
\end{array}\right] .
$$

Note that $\mathcal{M}_{0}(1,2), \mathcal{M}_{0}(2,3)$, and $\mathcal{M}_{0}(8,5)$ are the only " 1 " entries in their rows. In the first row, there are more " $\varnothing$ " elements. Following Step 1, we apply the reduction $\mathcal{M}_{1}=$

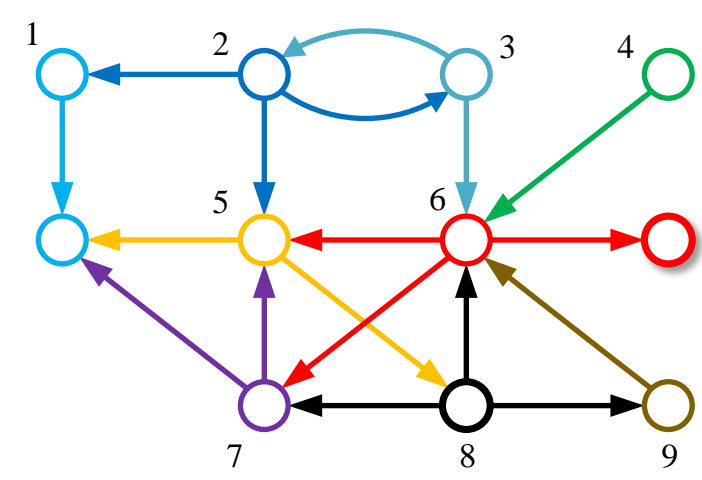

Fig. 3. A directed simple graph with 11 vertices, which is decomposed into 9 disjoint pseudo-trees, which are labeled with different colors.

$\mathscr{F}\left(\mathcal{M}_{0}, 1,2\right)$, which yields

$$
\mathcal{M}_{1}=\left[\begin{array}{llllllll}
0 & 1 & \varnothing & 0 & 0 & 0 & \varnothing & \varnothing \\
1 & 0 & 0 & \varnothing & 0 & \varnothing & 0 & 0 \\
\varnothing & 0 & 0 & \varnothing & 0 & \varnothing & 0 & 0 \\
0 & \varnothing & \varnothing & 0 & 1 & 0 & 0 & \varnothing \\
0 & 1 & 1 & 0 & 0 & 0 & 0 & 1 \\
0 & \varnothing & \varnothing & 0 & 0 & 0 & 1 & \varnothing \\
\varnothing & 0 & 0 & 1 & 0 & 0 & 0 & 0 \\
\varnothing & 0 & 0 & \varnothing & 0 & \varnothing & 0 & 0
\end{array}\right] \in \mathbb{M}^{8 \times 8}
$$

The resulting disjoint pseudo-tree covering is then given $\Pi_{1}=\left\{\mathcal{T}_{1}^{1}, \mathcal{T}_{2}^{1}, \cdots, \mathcal{T}_{8}^{1}\right\}$, where $\mathcal{T}_{1}^{1}=\mathcal{T}_{1}^{0} \cup \mathcal{T}_{2}^{0}$ and $\mathcal{T}_{i}^{1}=$ $\mathcal{T}_{i+1}^{0}$, for all $i=2,3, \cdots, 8$.

We continue to reduce the characteristic matrix using the same principle and obtain

$$
\begin{gathered}
\mathcal{M}_{2}=\mathscr{F}\left(\mathcal{M}_{1}, 1,2\right)=\left[\begin{array}{lllllll}
0 & 0 & 0 & 0 & 0 & 0 & 0 \\
0 & 0 & \varnothing & 0 & \varnothing & 0 & 0 \\
0 & \varnothing & 0 & 1 & 0 & 0 & \varnothing \\
0 & 1 & 0 & 0 & 0 & 0 & 1 \\
0 & \varnothing & 0 & 0 & 0 & 1 & \varnothing \\
0 & 0 & 1 & 0 & 0 & 0 & 0 \\
0 & 0 & \varnothing & 0 & \varnothing & 0 & 0
\end{array}\right], \\
\mathcal{M}_{3}=\mathscr{F}\left(\mathcal{M}_{2}, 3,4\right)=\left[\begin{array}{llllll}
0 & 0 & 0 & 0 & 0 & 0 \\
0 & 0 & 0 & \varnothing & 0 & 0 \\
0 & 1 & 0 & 0 & 0 & 1 \\
0 & \varnothing & 0 & 0 & 1 & \varnothing \\
0 & 0 & 0 & 0 & 0 & 0 \\
0 & 0 & 0 & \varnothing & 0 & 0
\end{array}\right],
\end{gathered}
$$

and

$$
\mathcal{M}_{4}=\mathscr{F}\left(\mathcal{M}_{3}, 4,5\right)=\left[\begin{array}{lllll}
0 & 0 & 0 & 0 & 0 \\
0 & 0 & 0 & 0 & 0 \\
0 & 1 & 0 & 0 & 1 \\
0 & 0 & 0 & 0 & 0 \\
0 & 0 & 0 & 0 & 0
\end{array}\right] \in \mathbb{M}^{5 \times 5},
$$

The last matrix $\mathcal{M}_{4}$ corresponds to the disjoint pseudo-tree covering $\Pi_{4}=\left\{\mathcal{T}_{1}^{4}, \mathcal{T}_{2}^{4}, \mathcal{T}_{3}^{4}, \mathcal{T}_{4}^{4}, \mathcal{T}_{5}^{4}\right\}$, where $\mathcal{T}_{1}^{4}=\mathcal{T}_{1}^{0} \cup$ $\mathcal{T}_{2}^{0} \cup \mathcal{T}_{3}^{0}, \mathcal{T}_{2}^{4}=\mathcal{T}_{4}^{0}, \mathcal{T}_{3}^{4}=\mathcal{T}_{5}^{0} \cup \mathcal{T}_{6}^{0}, \mathcal{T}_{4}^{4}=\mathcal{T}_{7}^{0} \cup \mathcal{T}_{8}^{0}, \mathcal{T}_{5}^{4}=\mathcal{T}_{9}^{0}$. The disjoint pseudo-tree covering $\Pi_{3}$ is illustrated in Fig. 4. 


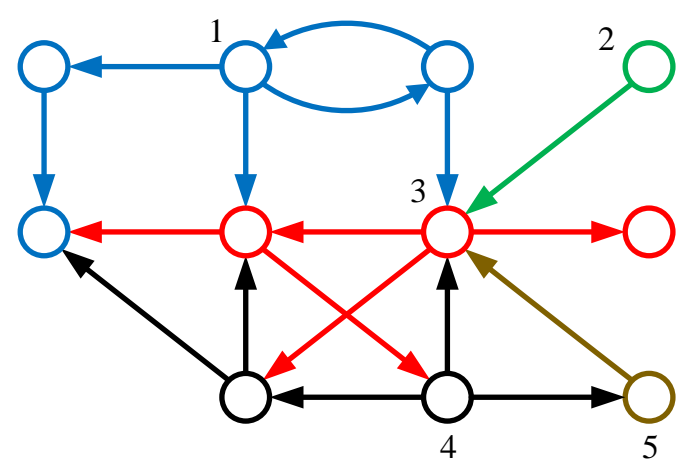

Fig. 4. After Step 1 of the proposed approach, the directed graph is decomposed into 5 disjoint pseudo-trees, labeled with different colors.

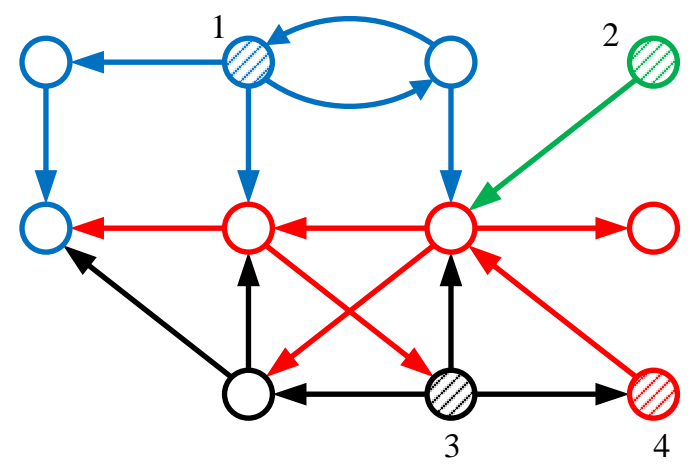

Fig. 5. The resulting disjoint pseudo-tree covering of the directed graph, where each pseudo-tree is labeled with a different color. One of the solution for allocating the excitation signals is to assign the shadowed vertices as the excited ones.

We continue to apply Step 2 to combine the remaining mergeable pseudo-trees in $\Pi_{4}$. Since the third row of $\mathcal{M}_{4}$ contains two "1" entries, we then take the following operation:

$$
\mathcal{M}_{5}=\mathscr{F}\left(\mathcal{M}_{4}, 3,5\right)=\left[\begin{array}{llll}
0 & 0 & 0 & 0 \\
0 & 0 & 0 & 0 \\
0 & 0 & 0 & 0 \\
0 & 0 & 0 & 0
\end{array}\right],
$$

which yields the disjoint pseudo-tree covering $\Pi_{5}=$ $\left\{\mathcal{T}_{1}^{5}, \mathcal{T}_{2}^{5}, \mathcal{T}_{3}^{5}, \mathcal{T}_{4}^{5}\right\}$, as shown in Fig. 5, with $\mathcal{T}_{1}^{5}=\mathcal{T}_{1}^{0} \cup \mathcal{T}_{2}^{0} \cup$ $\mathcal{T}_{3}^{0}, \mathcal{T}_{2}^{5}=\mathcal{T}_{4}^{0}, \mathcal{T}_{3}^{5}=\mathcal{T}_{5}^{0} \cup \mathcal{T}_{6}^{0} \cup \mathcal{T}_{9}^{0}$, and $\mathcal{T}_{4}^{5}=\mathcal{T}_{7}^{0} \cup \mathcal{T}_{8}^{0}$. (We may also do $\mathcal{M}_{4}=\mathscr{F}\left(\mathcal{M}_{4}, 3,2\right)$.) Then, we find that there does not exist mergeable pairs in $\Pi_{5}$ any more. Thus, four excitation signals are allocated at the roots of the pseudotrees for the generic identifiability of the overall network. One choice is to select the vertices labeled by shadowed vertices in Fig. 5.

\section{CONCLUSION}

In this paper, we have considered the allocation of external excitation signals for the generic identifiability of dynamical networks. A novel graph-theoretic condition of the generic identifiability has been provided, which relates to the disjoint pseudo-tree covering of the network. Based on the condition, we devise an efficient algorithm aiming to find the minimal number of excitation signals and their locations such that the network is generically identifiable.

\section{REFERENCES}

[1] M. Mesbahi and M. Egerstedt, Graph Theoretic Methods in Multiagent Networks. Princeton University Press, 2010.

[2] X. Cheng and J. M. A. Scherpen, "Clustering approach to model order reduction of power networks with distributed controllers," Advances in Computational Mathematics, vol. 44, no. 6, pp. 1917-1939, Dec 2018

[3] L. Ljung, System Identification: Theory for the User, ser. Prentice Hall information and system sciences series. Prentice Hall PTR, 1999.

[4] P. M. J. Van den Hof and R. J. Schrama, "Identification and control closed-loop issues," Automatica, vol. 31, no. 12, pp. 1751-1770, 1995.

[5] P. M. J. Van den Hof, A. Dankers, P. S. C. Heuberger, and X. Bombois, "Identification of dynamic models in complex networks with prediction error methods - basic methods for consistent module estimates," Automatica, vol. 49, no. 10, pp. 2994-3006, 2013.

[6] A. Chiuso and G. Pillonetto, "A bayesian approach to sparse dynamic network identification," Automatica, vol. 48, no. 8, pp. 1553-1565, 2012.

[7] D. Materassi and M. V. Salapaka, "On the problem of reconstructing an unknown topology via locality properties of the wiener filter," IEEE Transactions on Automatic Control, vol. 57, no. 7, pp. 1765-1777, 2012.

[8] A. Dankers, P. M. J. Van den Hof, X. Bombois, and P. S. C. Heuberger, "Identification of dynamic models in complex networks with prediction error methods: Predictor input selection," IEEE Transactions on Automatic Control, vol. 61, no. 4, pp. 937-952, 2016.

[9] K. R. Ramaswamy, G. Bottegal, and P. M. J. Van den Hof, "Local module identification in dynamic networks using regularized kernelbased methods," in 2018 IEEE Conference on Decision and Control (CDC). IEEE, 2018, pp. 4713-4718.

[10] M. Gevers and A. S. Bazanella, "Identification in dynamic networks: identifiability and experiment design issues," in Proceedings of 54th IEEE Conference on Decision and Control (CDC). IEEE, 2015, pp. 4005-4010.

[11] M. Gevers, A. S. Bazanella, and G. V. da Silva, "A practical method for the consistent identification of a module in a dynamical network," IFAC-PapersOnLine, vol. 51, no. 15, pp. 862-867, 2018.

[12] A. S. Bazanella, M. Gevers, J. M. Hendrickx, and A. Parraga, "Identifiability of dynamical networks: which nodes need be measured?" in Proceedings of IEEE 56th Annual Conference on Decision and Control (CDC). IEEE, 2017, pp. 5870-5875.

[13] H. H. M. Weerts, P. M. J. Van den Hof, and A. G. Dankers, "Identifiability of linear dynamic networks," Automatica, vol. 89, pp. 247-258, 2018.

[14] J. M. Hendrickx, M. Gevers, and A. S. Bazanella, "Identifiability of dynamical networks with partial node measurements," IEEE Transactions on Automatic Control, vol. 64, no. 6, pp. 2240-2253, June 2019

[15] H. J. van Waarde, P. Tesi, and M. K. Camlibel, "Necessary and sufficient topological conditions for identifiability of dynamical networks," arXiv preprint arXiv:1807.09141, 2018.

[16] H. Weerts, P. M. J. Van den Hof, and A. Dankers, "Single module identifiability in linear dynamic networks," in Proceedings of 57th IEEE Conference on Decision and Control. IEEE, 2018, pp. 47254730.

[17] C. Godsil and G. F. Royle, Algebraic Graph Theory. Springer Science \& Business Media, 2013, vol. 207.

[18] J. Nikiel, Topologies on Pseudo-trees and Applications. American Mathematical Society, 1989, vol. 416.

[19] D. Cvetković and P. Rowlinson, "Spectra of unicyclic graphs," Graphs and Combinatorics, vol. 3, no. 1, pp. 7-23, 1987. 